Anu Ormisson-Lahe, Kristiina Tiideberg

\title{
CARL JULIUS SENFF (1804-1832) \\ ALS ARCHITEKT UND GRAFIKER. ERGÄNZUNGEN ZUR BAUGESCHICHTE DER UNIVERSITÄT ZU TARTU (DORPAT)
}

Über Carl Julius Senff (1804-1832), den Sohn des Zeichenlehrers der Kaiserlichen Universität zu Dorpat (est. Tartu) Carl August Senff (1770-1838), hat bis jetzt der deutsche Kunstwissenschaftler Gerd-Helge Vogel den gründlichsten Überblick geliefert. ${ }^{1}$ Vogel konzentriert sich in seinen Aufsätzen auf Senffs späteres Werk, während gleichzeitig über die Entwicklungsjahre des jungen, begabten Künstlers, von dem erhofft wurde, der Nachfolger des Universitätsarchitekten Johann Wilhelm Krause zu werden, in der Dorpater Zeit und seine Aktivitäten an der Universität bis jetzt wenig bekannt ist. Das Ziel des vorliegenden Beitrags besteht darin, diese Lücke zu füllen und vor allem die Arbeiten seiner frühen Dorpater Zeit zu markieren. Einen wichtigen Anstoß zum Verfassen dieses Aufsatzes lieferten aber zwei kürzlich aufgefundene architektonische

DOI: https://doi.org/10.12697/BJAH.2019.18.07

Übersetzung aus dem Estnischen von Marju und Olaf Mertelsmann.

1 Gerd-Helge Vogel, „Carl Julius Senff - üks lühike kunstnikuelu“, Eesti Kunsti Ajalugu, 3: 1770-1840, hrsg. von Juhan Maiste (Tallinn: Eesti Kunstiakadeemia 2017), 222-225; GerdHelge Vogel, „Carl Julius Senff (1804-1832) - ein Kurzes Künstlerleben als Architekt, Zeichner und Grafiker", Baltic Journal of Art History, 14 (2017), 95-114. 


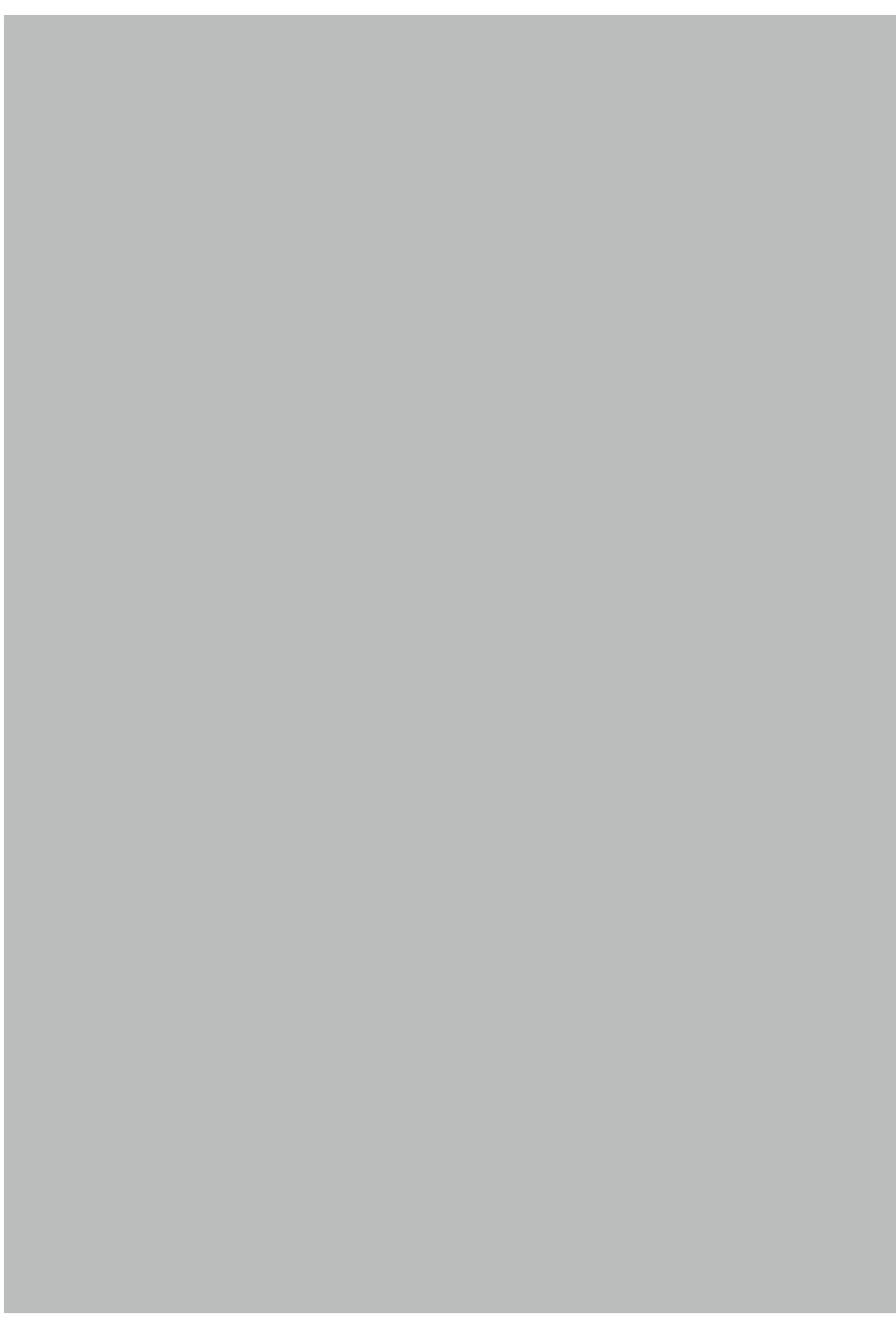

ABB. 1. AUGUST FERDINAND HOPFGARTEN (1807-1896), CARL JULIUS SENFFS PORTRAIT BLEISTIFTZEICHNUNG, WAHRSCHEINLICH 1831-1832. METROPOLITAN MUSEUM OF ART.
Zeichnungen, die der jungen Carl Julius Senff für das Ensemble der Universität zu Dorpat entwarf.

Carl Julius Senff (Abb. 1) ist als einer der begabtesten Schüler seines Vaters bezeichnet worden, über den wir wegen seines frühen Todes und der kurzen Zeit seines Schaffens leider nur wenig wissen. C. J. Senff kam am 23. Dezember (nach dem alten Kalender am 11 Dezember) 1804 in Dorpat als drittes Kind seiner Eltern zur Welt. Nach den Schuljahren am Gouvernementsgymnasium in Dorpat begann er seine künstlerische Ausbildung unter Anleitung seines Vaters. Sein Vater vermittelte ihm eine Einleitung in das Zeichnen und lehrte ihn verschiedene grafische Techniken. Im Jahr 1823 nahm ihn der Vater damals schon als Student der Universität zu Dorpat mit nach St. Petersburg, damit er dort Professor Johann Friedrich Erdmann beim Lithografieren von Portraits hilft. In Dorpat gab es zu dieser Zeit noch keine lithografische Werkstatt, doch diese neue Technik wurde zunehmend populär und C. A. Senff reiste nach St. Petersburg, um dort seine ersten Lithografien umzusetzen. ${ }^{2}$ Es erscheint als möglich dass der Sohn dort erstmals erfolgreich die Lithografie ausprobierte, dies wird durch ein im Juli 1823 geschaffenen Portrait eines Mannes belegt, das in der Sammlung des Kunstmuseums Tartu aufbewahrt wird (Abb. 2). Das Portrait, das die Unterschrift nach Gröger trägt, wurde offensichtlich nach dem deutschen Künstler Friedrich Carl Gröger (1766-1838) angefertigt oder kann dessen (Selbst-) Portrait darstellen. Die Arbeit zeigte die Reife des erst 19-jährigen Künstlers als Zeichner und Grafiker. Später hat Carl Julius Senff noch mehrere lithografische Portraits umgesetzt, von denen das bekannteste das Portrait des Architekten Carl Ludwig Engel (1829) in der Sammlung des Sinebrychoff-Museums ist (Abb. 4). Im Stadtarchiv Halle wird dagegen das Portrait eines unbekannten Soldaten (1828; Abb. 3) aufbewahrt.

Während seiner Studienzeit trat auch sein Interesse für Architektur und Kunsttheorie auf. 1825 erschien das von C. J. Senff herausgegebene Lehrbuch der Perspektive für diejenigen Landschaftsmaler, die sich ihre Kenntnisse selbst aneignen (Perspective für Landschaftsmaler

2 Zusätzlich zum Portrait von Professor Erdmann (Universitätsbibliothek Tartu, ÜR 4359) st wenigstens eins von Senff im Jahr 1823 erschaffenes lithografische Portrait bekannt, sieh Voldemar Vaga, Kunst Tartus XIX sajandil (Tallinn: Kunst, 1971), 13. 


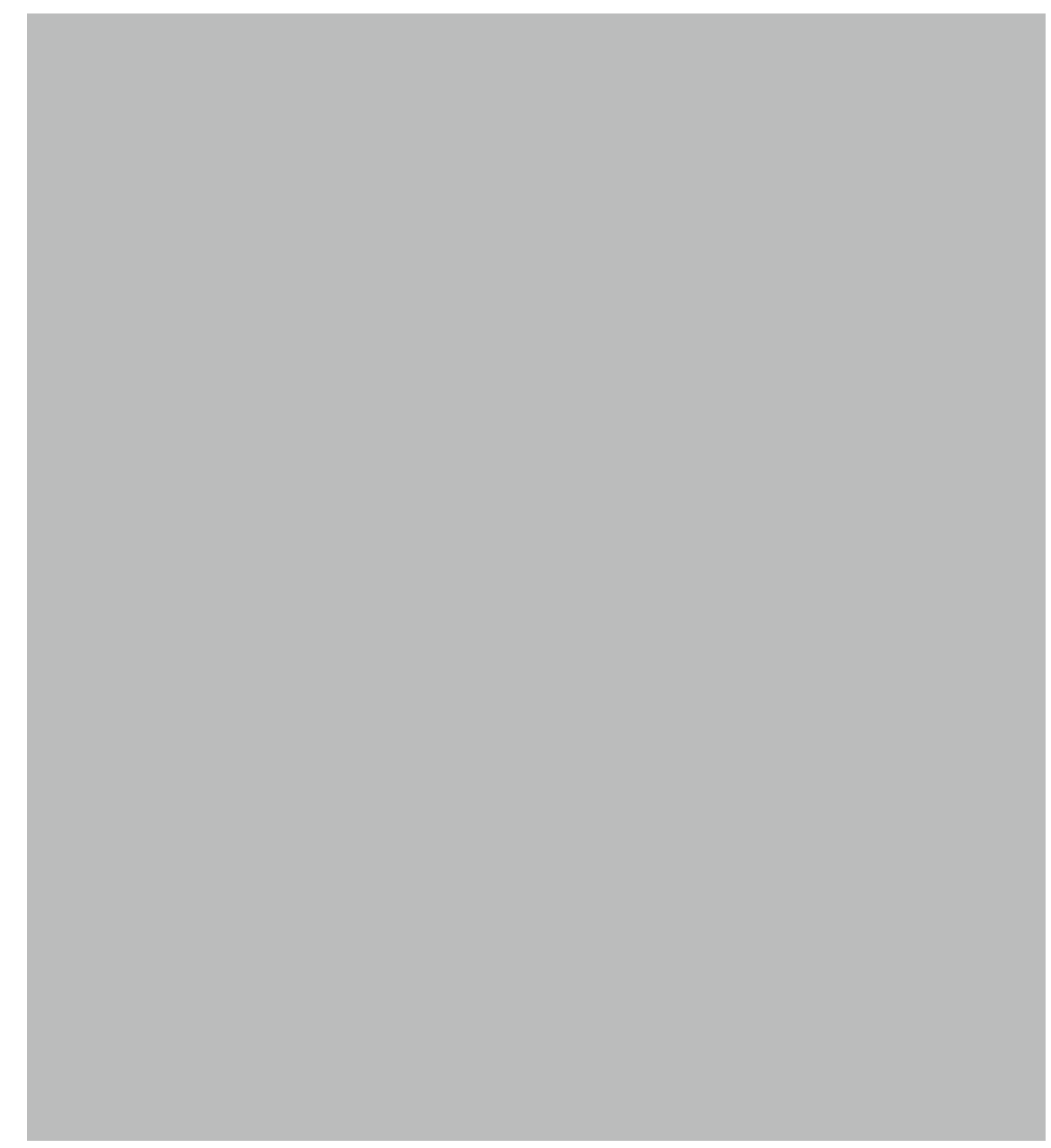

ABB. 2. CARL JULIUS SENFF, NACH FRIEDRICH CARL GRÖGER (?), PORTRAIT EINES UNBEKANNTEN MANNES. LITHOGRAFIE, 1823. KUNSTMUSEUM TARTU.

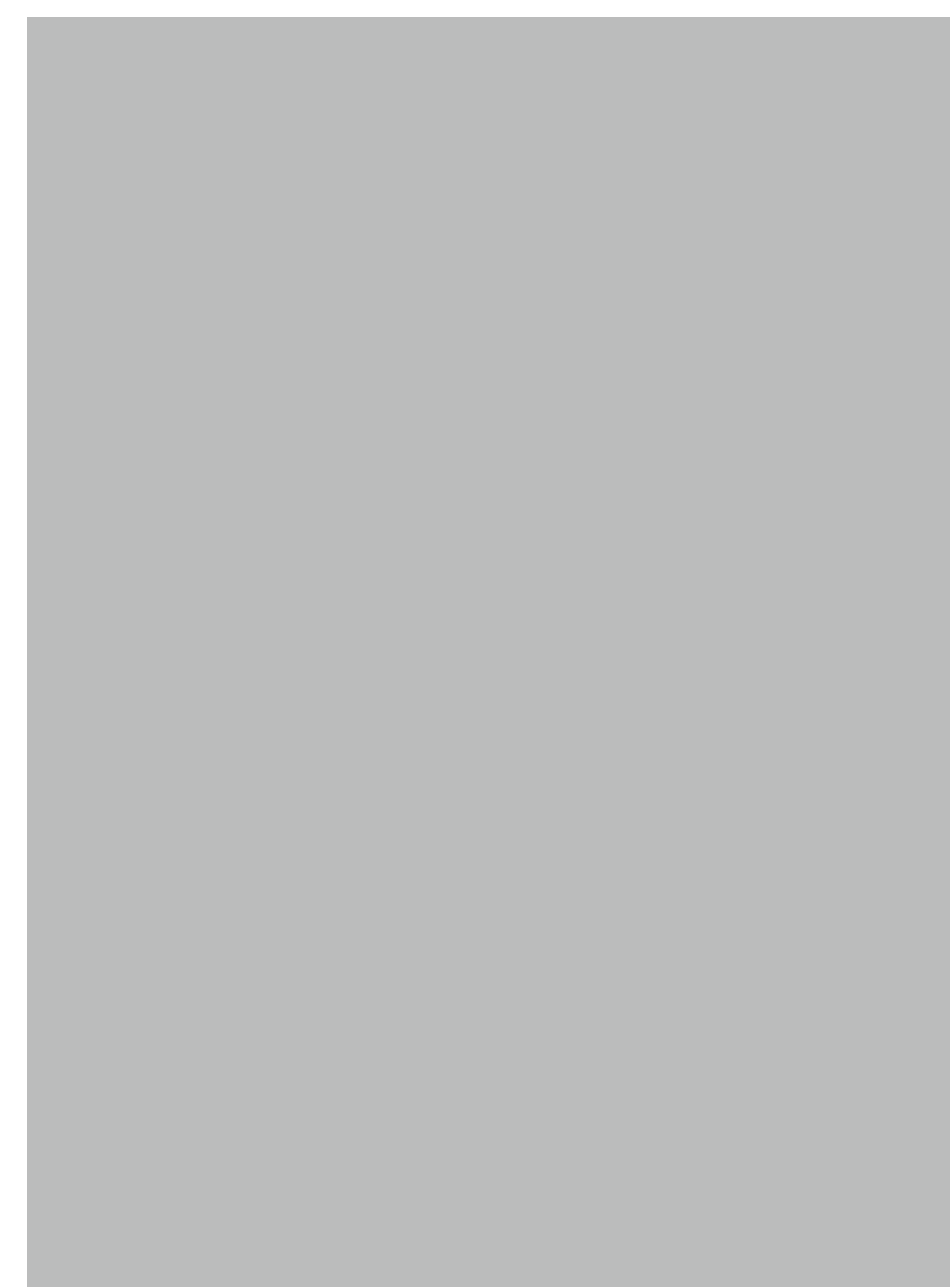

ABB. 3. CARL JULIUS SENFF, PORTRAIT EINES UNBEKANNTEN JUNGEN MANNES. LITHOGRAFIE 1828. STADTARCHIV HALLE. 


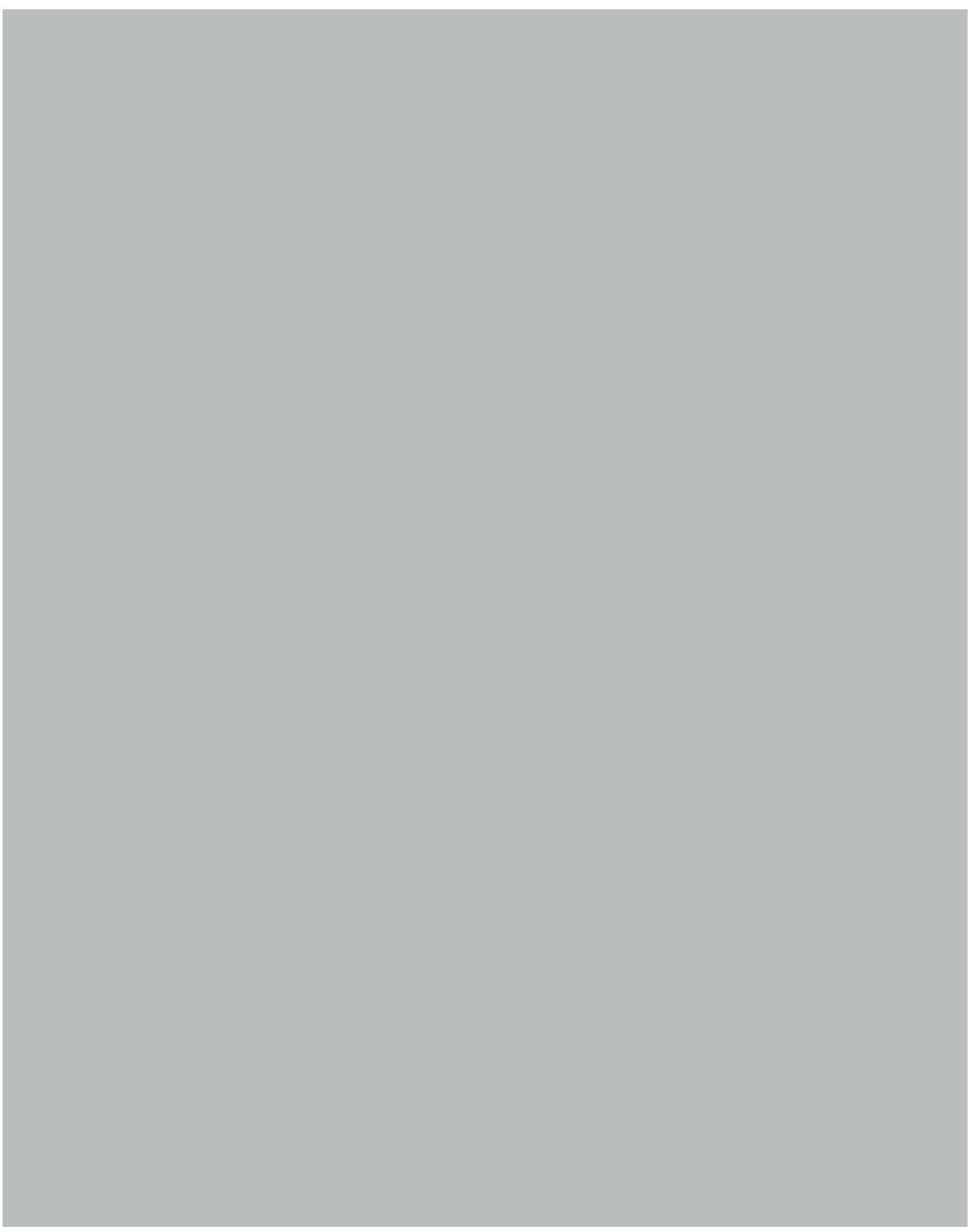

ABB. 4. CARL JULIUS SENFF, PORTRAIT DES ARCHITEKTEN CARL LUDWIG ENGEL. LITHOGRAFIE, 1829. SINEBRYCHOFF-KUNSTMUSEUM.

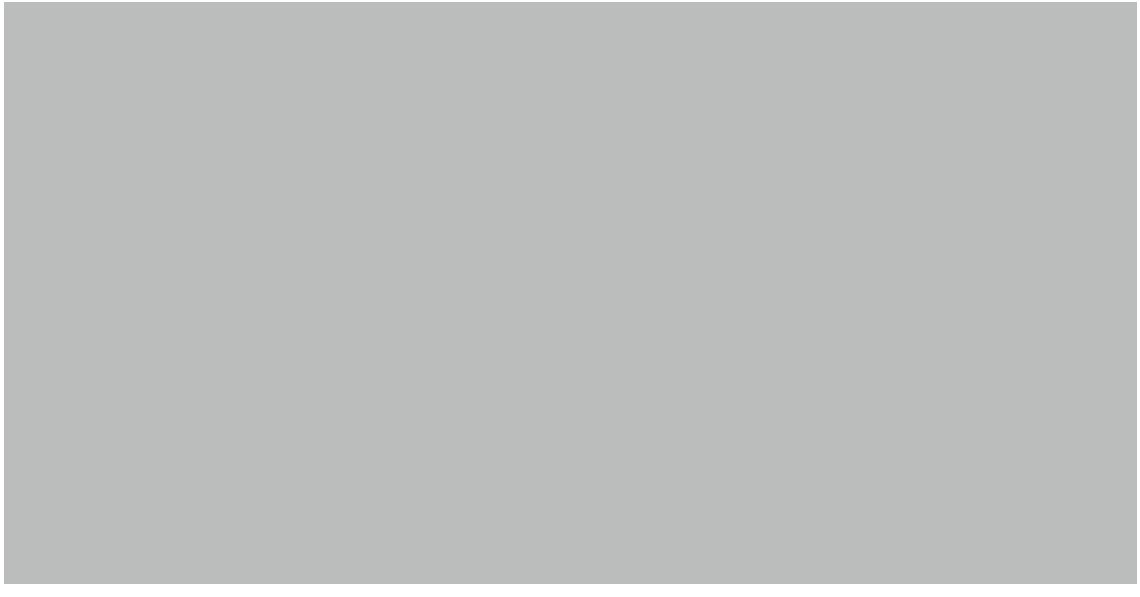

ABB. 5. CARL JULIUS SENFF, ILLUSTRATION FÜR DAS VON IHM VERFASSTE LEHRBUCH PERSPECTIVE FÜR LANDSCHAFTSMALER ZUM SELBSTSTUDIUM (DORPAT: BEI A. STICINSKY, 1825).

zum Selbststudium, 1825). ${ }^{3}$ Auf diese Weise folgte Julius dem Vorbild seines Vaters, der während seiner Tätigkeit an der Universität ebenso Lehrmaterialien auf dem Gebiet erstellte. ${ }^{4}$ Diese Ausgabe enthält zwei von Senff gravierte Illustrationen (Abb. 5). 1827 erhielt C. J. Senff eine Silbermedaille für eine Arbeit auf dem Gebiet der räumlichen Geometrie, die zwei Jahre später auf Kosten der Universität als Publikation erschien (Systematische Darstellung der Hauptsätze der Geometrie im Raume, 1829).

Nach Abschluss der Universität Dorpat im Jahr 1827 reiste C. J. Senff zur weiteren Fortbildung zum Stadtbaumeister von Helsinki (schw. Helsingfors), Carl Ludwig Engel, ${ }^{5}$ der sich in dieser Zeit

3 Auf der Rückseite des Titels tritt zutage, dass zu diesem Zeitpunkt Johann Wilhelm Krause das Amt des Universitătszensors bekleidete. „Der Druck dieser Schrift wird unter der Bedingung bewilligt: das nach dem Abdrucke und vor der Herausgabe derselben, sieben Exemplare an das Censur-Commiteé der Kaiserl. Universität allhier zur vorschriftmässigen Vertheilung eingesende werden. Dorpat, den 1. Novbr. 1824. Professor Joh. Wilh. Krause, Censor.“

4 Siehe zum Beispiel: Juta Keevallik, Kristiina Tiideberg, „Karl August Senff - ülikool joonistusõpetaja ja kunstnik“" Eesti Kunsti Ajalugu, 3: 1770-1840, hrsg. von Juhan Maiste (Tallinn: Eesti Kunstiakadeemia 2017), 211-215.

5 Voldemar Vaga, Tartu ülikooli arhitektid (Tartu: Akadeemiline Kirjandusühing, 1928), 25 Vogel, „Carl Julius Senff - üks lühike kunstnikuelu“, 222. 
hauptsächlich auf den Ausbau des Universitätsensembles von Helsinki konzentrierte. Die 1827 eingerichtete Universität von Helsinki sollte genauso wie Senffs Heimatuniversität der "Verschönerung der Stadt" dienen $^{6}$ und dies bot dem jungen, an Architektur interessierten Mann die Möglichkeit, in der Praxis eine wichtige Arbeit eines damals bereits bekannten Architekten zu verfolgen. Die Hingabe für architektonische Studien durch Carl Julius Senff wurde bereits an der Universität Dorpat bemerkt und als Johann Wilhelm Krause im Jahr 1828 verstarb, dann wurde gehofft, dass gerade er als neue Lehrkraft für Baukunst ausgebildet werden kann. ${ }^{7}$ Die Universität beantragte für den jungen Senff ein dreijähriges kaiserliches Stipendium zur Fortbildung im Ausland und verband dies mit der Bedingung, dass er im Falle seiner Wahl zum Professor für wenigstens sechs Jahre als Lehrkraft an der Universität Dorpat arbeiten sollte. Im Juni 1829 begab er sich auf den Weg nach Deutschland, er reiste nach Berlin, Dresden, Sachsen, Jena, Prag und Wien. Von diesen Reisen ist durch Glück auch eine Anzahl von Zeichnungen erhalten geblieben (die sich hauptsächlich in der Sammlung des Estnischen Kunstmuseums befinden), darüber hat Vogel bereits gründlich geschrieben. ${ }^{8} \mathrm{Im}$ Jahr 1830 erwarb Carl Julius Senff in Jena den Titel eines Doktors der Philosophie. 1831 erschien in Berlin sein bekanntestes Werk, eine zehnblättrige Serie von Ansichten und Zeichnungen des Veitsdoms in Prag, ausgeführt in der Aquatintentechnik. ${ }^{9}$ In dieser Serie tritt sein großes Interesse an gotischer Baukunst zutage, die bereits in den Jahren in Dorpat angefangen haben könnte, als die Pläne für den Wiederaufbau der Domkirche in den 1820er Jahren ebenfalls hauptsächlich von der Neogotik ausgegangen waren. ${ }^{10}$ Am Ende

6 Kalevi Pöykko, „Das Hauptgebäude der Kaiserlichen Alexander-Universität von Finnland. Eine Untersuchung der Entwurfsstadien und Baugeschichte des Gebäudes von Carl Ludwig Engel mit Klärung des Stil- und ideengeschichtlichen Hintergrunds und Erläuterung der Kompositionsmittel", Soumen Muinaismuistoyhdistyksen Aikakaukirja / Finska Fornminnesföreningens Tidskrif (Helsinki, 1971), 31-32.

7 Vogel, „Carl Julius Senff - üks lühike kunstnikuelu“, 222.

8 Gerd-Helge Vogel, „Carl August Senff (1770-1838) im Kontext der Deutschen Kunst und sein Wirken in Tartu (Dorpat)“, Baltic Journal of Art History, 14 (2017), 53-93.

9 Abteilung für Handschriften und seltene Bücher der Universitätsbibliothek Tartu [Tartu ülikooli raamatukogu, TÜR], ÜR 1869, 6695-6697, 8961-8965.

10 Zur Wiedergeburt der Domkirche und der Gotik siehe ausführlicher: Juhan Maiste, Anu Ormisson-Lahe, „Toomkirik valgustusajastu valgusel. Varemetest kerkib raamatukogu“, Tartu toomkirik. Katedraal. Raamatukogu. Munseum, hrsg. von Mariann Raisma, Krista Andreson (Tartu: Tartu Ülikooli muuseum, 2017), 110ff. des Jahres 1831 reiste er nach Italien, wo er unerwartet am 19. April 1832 in Mailand verstarb.

Obwohl Carl Julius Senff bereits zu Studienzeiten über ein großes Interesse an Architektur verfügte und er sich sowohl in Helsinki bei Engel als auch auf Reisen in Europa fortbildete, gelang es ihm nicht, als praktizierender Architekt aktiv zu werden. Deswegen sind die erst kürzlich im Russischen Staatlichen Historischen Archiv in St Petersburg aufgefundenen Zeichnungen bemerkenswert. Es handelt sich um die einzigen bekannten Bauentwürfe von Carl Julius Senff (Abb. 6 und 7), welche die Fassaden, Schnitte, den Grundriss und den Lageplan des bei der Sternwarte in Dorpat geplanten Wohnhauses des Observators darstellten. ${ }^{11}$ Es fehlt eine Datierung auf den Zeichnungen, doch es kann vermutet werden, weil Senff Dorpat im Frühjahr 1829 verließ, dass sie zuvor angefertigt worden waren. Im Russischen Staatlichen Historischen Archiv sind sie auf das Jahr 1824 datiert worden, ${ }^{12}$ dasselbe Jahr in dem auf Initiative von Professor Georg Friedrich Parrot die Planung für eine neue drehbare Kuppel für die Sternwarte begonnen wurde. ${ }^{13}$ Diese Datierung würde bestätigen, dass Überlegungen für den Bau einer Wohnung für den Observator bereits in den 1820er Jahren bestanden, also früher, als bisher vermutet. ${ }^{14}$ Andererseits kann die Anfertigung der Zeichnungen auch in spätere Jahre verortet werden (1825-1827), als Carl Julius Senff mit der Illustrierung von Publikationen der Sternwarte verbunden war (siehe ausführlicher unten). In diesem Zusammenhang erscheint es als interessant zu bemerken, dass obwohl die Pläne sämtlicher Universitätsgebäude (sowohl der fertiggestellten als auch der nur geplanten) aus den Jahren 1800 bis 1831 dem Universitätsarchiv

11 Die Zeichnungen befinden sich im Russischen Staatlichen Historischen Archiv [Rossijskij Gosudarstvennij Istoričeskij Archiv, RGIA] in St. Petersburg und für deren Nutzung danken die Autoren Georgij Smirnov, der diese Zeichnungen im Rahmen seiner Archivarbeit fand. Die Zus B1. 1 und 2, vom Archiv af das Jahr 1824 datiert.

12 Archivbeschreibung: Universität in der Stadt Derpt. Projekt von Wohnraum für da Observatorium, mit den entsprechenden Wohnungen für Professoren und Observatoren. Fassade, Durchschnitt, Pläne des Gebäudes und des Orts. 1824, http://rgia.su/object/773127041 (eingesehe am 03.09.2019).

13 Nationalarchiv [Rahvusarhiiv, RA], EAA.402.5.186, Bl. 1-3. Siehe auch Epi Tohvri, Georges Frédéric Parrot. Tartu keiserliku ülikooli esimene rektor (Tartu: Tartu Ülikooli kirjastus, 2019), $1007 \mathrm{ff}$.

14 Siehe auch die entsprechenden Gesuche des Direktors der Sternwarte Struve in den Jahren 1833 und 1837, ERA.T-76.1.2081; Elsbet Parek, Tartu tähetorni ajalooline õiend (1974), 26-27. 

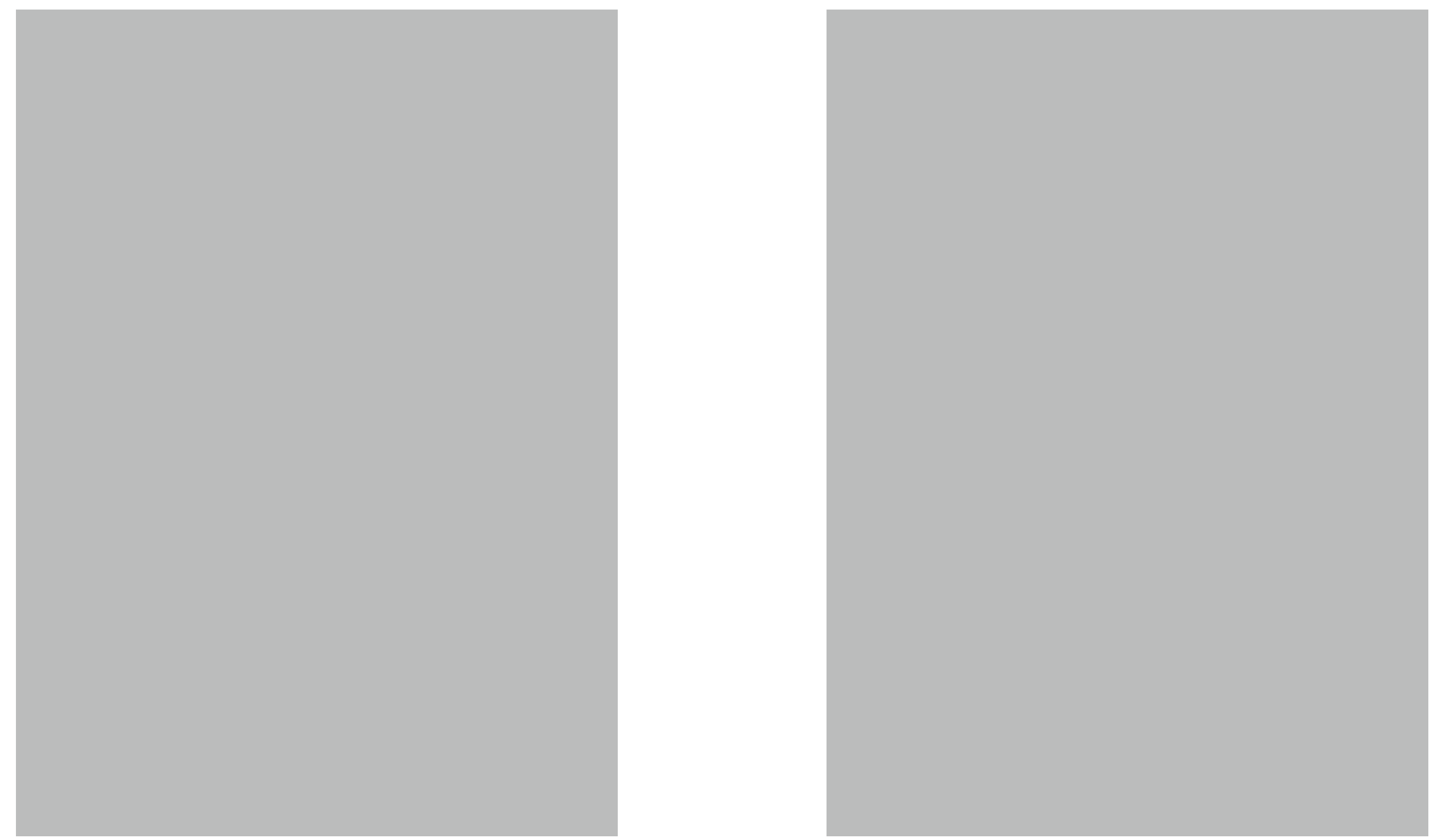

ABB. 7. CARL JULIUS SENFF, LAGEPLAN UND DURCHSCHNITT DES OBSERVATORIUMS RUSSISCHES STAATLICHES HISTORISCHES ARCHIV. 
übergeben wurden und darüber auch im Jahr 1834 eine exakte Liste angefertigt wurde ${ }^{15} \mathrm{im}$ Bestand fehlen dennoch der Entwurf für die Wohnung des Observators und der Name von Senff.

Die von Carl Julius Senff angefertigten Zeichnungen sind ausgeführt worden, mit einer Überschrift versehen (PROJECT zu einer Wohnung des Observators bei der Sternwarte der kaiserl Univ. DORPAT) und signiert (unten rechts gezeichnet von J. Senff). Auf der ersten Bauplan (Abb. 6), auf der die Signatur zur Hälfte zerstört ist, wird die Fassade des Hauses angeführt (Facade gegen Osten) und der Grundriss sowie unten in der Mitte der Maßstab. Auf der kolorierten Zeichnung ist ein einstöckiges Gebäude mit einem niedrigen Walmdach zu sehen, dessen Seitenrisalite aus Ziegelstein geplant sind. Die Seitenrisalite sind mit Triglyphen dekoriert und den für die Gebäude der Universität Dorpat typischen Zahnkarniesen. Auf dem Grundriss wird die Raumaufteilung dargestellt, die mit den Buchstaben von a bis i markiert sind, doch leider ist die exakte Erklärung der Räume unbekannt. Für das Gebäude sind auch zwei Schornsteine und vier Feuerstellen geplant, von denen zwei offensichtlich die größeren Räume erwärmen sollen, die möglicherweise für die Nutzung als Wohnzimmer oder als Saal gedacht sind. Auf der linken Seite der Zeichnung vom Raum h aus führt offenbar eine Kellertreppe herunter. Für das Gebäude sind zwei Eingänge geplant, von denen einer mit einer einfachen Treppe wahrscheinlich in den für die Dienerschaft gedachten Hausteil führt, eine zweite großanlegte Treppe befindet sich aber vor dem Haupteingang. Im oberen Teil der zweiten Zeichnung (Abb. 7) wird der Lageplan des Gebäudeensembles der Sternwarte dargestellt, bei dem zusätzlich zur von Johann Wilhelm Krause erbauten Sternwarte auch das Haus des Astronomen (auf der Zeichnung als Wohnung des Professors markiert) zusammen mit dessen Hilfsgebäude und auf der Nordseite der Sternwarte das Haus des Observators (Wohnung des Observators) abgebildet sind. Das von der Nordfassade der Sternwarte etwas links geplante Haus des Observators befindet sich ziemlich am Rand der ehemaligen Bastion. Der im unteren Teil der Zeichnung abgebildete Querschnitt des Hauses des Observators zeigt deutlich die Unterschiede der Bodenoberfläche an, denn der gewölbte Keller ist nur für den halben

15 RA, EAA.402.5.282, publiziert in: Johann Wilhelm Krause 1757-1828: kataloog. 4, Ülikool Emajõe Ateenas = Katalog 4. Die Universität im Embach-Athen, hrsg. von Anu Ormisson-Lahe (Tartu; Tallinn: Tartu Ülikool; Eesti Keele Sihtasutus, 2016), 484ff.
Umfang des Gebäudes geplant. Auf dem Querschnitt des einfachen, einstöckigen Hauses sind Fundament, Treppe, Dachkonstruktion und Schornstein ersichtlich. Auf dem Querschnitt ist erkennbar, dass die im Gebäude befindlichen Räume sich etwas in der Höhe unterscheiden. Vom Lageplan aus ergibt sich, dass für das Haus zwei Vorräume geplant waren, einer auf der nördlichen, der andere auf der westlichen Seite. Im letzteren Fall ist auf der Querschnittszeichnung deutlich zu sehen, dass es sich um ein repräsentatives Portal handelt, zu dem eine achtstufige Treppe führt. Das einfache Wohnhaus des Observators ist mit gutem Geschmack und harmonisierend mit dem Ensemble der Sternwarte und mit den übrigen Universitätsgebäuden entworfen worden. Der junge Senff zeigt auf seiner einzigen bekannten architektonischen Zeichnung eine gute Detailkenntnis, ebenso wie eine Erfassung des Ganzen, was auch charakteristisch war für Krause, der in den prägenden Jahren von Senff das Gebäudeensemble der Universität errichtet hatte.

Der Direktor der Sternwarte Friedrich Georg Wilhelm Struve (1793-1864, seit 1831 von Struve, Direktor der Sternwarte in Dorpat 1820-1839) betonte laut der heute bekannten Archivquellen die Notwendigkeit des Baus einer Wohnung für den Observator in den Jahren $1833^{16}, 1837^{17}$ und 1839, sein Nachfolger Dr. Johann Heindrich Mädler auch noch im Jahr $1841^{18}$. Als Begründung für den Bau einer gesonderten Wohnung für den Observator wurde die räumliche Enge in der Sternwarte angeführt, weiterhin wurde betont, dass der Observator der Sternwarte möglichst nahe bei ihr wohnen sollte, um für beständige Messungen zu ihr nur einen kurzen Weg gehen zu müssen. Besonders betonte Struve in seinem Schreiben von 1837, dass sie alle in einem harten Klima lebten und es dann verantwortungslos sei, sich nicht um die Gesundheit des Observators zu sorgen..$^{19}$ Vom Februar 1837 bis zum September 1841 bestand auch ein aktiver Schriftverkehr mit dem Kurator des Lehrbezirks zur Frage des Baus einer Wohnung für den Observator, ${ }^{20}$

16 RA, EAA.402.5.224, B1. 182-184v.

17 RA, EAA.402.5.224, Bl. 209.

18 RA, EEA.402.5.224, Bl. 246-246v.

19 RA, EEA, 402.5.224, Bl. 209.

20 RA, EAA.381.1.687, Briefwechsel mit dem Ministerium der Volksaufklärung und dem Direktor der Sternwarte der Dorpater Universität über den Bau einer Amtswohnung für den
Observator. 
doch damals war geplant, den Entwurf des Hauses bereits bei Moritz Hermann Jacobi in Auftrag zu geben, ${ }^{21}$ der frühere Entwurf von Carl Julius Senff wurde nicht erwähnt. Doch das Gebäude wurde offensichtlich aus wirtschaftlichen Gründen nicht erbaut, denn 1836 hatte die Universität einen neuen Kurator erhalten, Gustav von Craffström, der Bau- und Renovierungsprojekten sehr kritisch gegenüberstand und neue Bauprojekte, darunter eine Universitätskirche, die Jacobi bereits entworfen hatte, wurden nicht in Angriff genommen. Eine gewisse Rolle beim Steckenbleiben der Baupläne spielte offenbar auch Struves Abgang aus Dorpat 1839. ${ }^{22}$ Deshalb wurde auch scharfsinnig vermerkt, dass der Plan der Struve-Zeit, für den Observator in der Nähe der Sternwarte ein Haus zu bauen, dazu führte, dass 1865 anstelle des Hauses eine abgesonderte Latrine errichtet wurde. ${ }^{23}$

Der Entwurf eines Wohnhauses für den Observator der Sternwarte der Universität Dorpat blieb nicht die einzige Arbeit von Senff, die in Verbindung mit der Sternwarte stand. Bereits während des Studiums illustrierte er mehrere von Struve zusammengestellte Publikationen. Im Jahr 1825 erschien eine Beschreibung von Struve über das kürzlich für die Sternwarte erworbene Fraunhofer-Teleskop (Beschreibung des auf der Sternwarte der Kaiserlichen Universität zu Dorpat befindlichen grossen Refractors von Fraunhofer, 1825). Ein Fraunhofer-Teleskop war damals das größte und beste Teleskop in der Welt, sein Erwerb stellte ein großes Verdienst für Struve und die Universität dar. Wegen des Teleskops musste auch die Kuppel der Sternwarte umgebaut werden, was zum großen Teil nach den Entwürfen des Rektors Georg Friedrich Parrot erfolgte. In den Publikationen finden sich vier Illustrationen in der Technik der Aquatinta und der Radierung: drei stellen das neue Teleskop dar (Abb. 8) und eine die umgebaute Kuppel der Sternwarte, für die letztere dienten offenbar die Entwürfe Parrots vom Dezember 1824 als Vorlage. ${ }^{24}$ Für Struve hat Carl Julius Senff noch wenigstens einmal Illustrationen erschaffen: für die 1827

21 RA, EAA.381.1.687, Bl. 2ff.

22 Tartu tähetorn $=$ Tartu Old Observatory, hrsg. von Lea Leppik (Tallinn: Aasta Raamat, 2011), 68 .

23 Ibidem, 74.

24 Eda Tursk, „Tähetorn“, Johann Wilhelm Krause 1757-1828: kataloog. 3. Linnaehitajana Tartus $=$ Katalog. 3. Als Stadtbauer in Tartu, hrsg. von Anu Ormisson-Lahe (Tartu: Tart Ulikool, 2011), 207-209.

ABB. 8. CARL JULIUS SENFF, DER GROSSE FRAUNHOFER-REFRAKTOR. RADIERUNG, AQUATINTA 1825. ILLUSTRATION FÜR DIE VERÖFFENTLICHUNG: F. G. W. STRUVE, BESCHREIBUNG DES AUF DER STERNWARTE DER KAISERLICHEN UNIVERSITAT ZU DORPAT BEFINDLICHEN GROSSEN 
erschienene Publikation der ersten Messergebnisse von Struves geodätischem Bogen. ${ }^{25}$ Darin ist eine von Senff gravierter Plan der von Jakobstadt (lett. Jēkabpils) bis zur Insel Hogland (russ. Gogland) reichenden Triangulationskette, in deren Mittelpunkt die Sternwarte in Dorpat liegt. Bei der Illustrierung dieser Werke lieferte erneut sein Vater offenbar einen wichtigen Anstoß, der als Kupferstecher und Zeichenlehrer tätig war und ebenso umfangreich als Illustrator der Publikationen der Universität.

Mit der Sternwarte war die Familie Senff auch eng verbunden. Carl August Senff wurde auf Empfehlung des Gatten der Schwester seiner Ehefrau, Ernst Christoph Friedrich Knorre (1759-1810) als Zeichenlehrer nach Dorpat eingeladen. Knorre war in Dorpat der erste Astronom und ein außerordentlicher Professor (18021810). Nach Knorres Tod kümmerte sich Senff um dessen Sohn, Karl Friedrich Knorre, der zusammen mit Struve gearbeitet und ein auffälliges Talent gezeigt hatte. Später erhielt Karl Knorre auf Empfehlung von Senff den Posten eines Astronomen der russischen Schwarzmeerflotte. Er baute in Zusammenarbeit mit Admiral Aleksej Greig das Meeresobservatorium in Nikolaev (heute Mykolaiv in der Ukraine) auf, war dessen erster Leiter ${ }^{26}$ und arbeitete dort insgesamt 51 Jahre lang. Nach der Abreise Struves aus Dorpat war für einige Zeit der Bruder von Julius Senff für die Sternwarte in Dorpat zuständig, der Mathematiker Carl Eduard Senff (1810-1849, kommissarischer Direktor der Sternwarte 1839-1840).

Der unerwartete Tod von Carl Julius Senff führte dazu, dass der Posten des Universitätsarchitekten nach dem Tod Krauses bis zum Jahr 1835 nicht besetzt wurde. Erst dann traf Hermann Moritz Jacobi ein. Julius Senffs Hinterlassenschaft besteht zusätzlich zu den im Ausland angefertigten Zeichnungen und den wenigen veröffentlichten Illustrationen auch im außergewöhnlich durchkomponierten Entwurf des Wohnhauses des Observators, welches die Sternwarte und das Haus des Astronomen zu einer einheitlichen Ganzheit verbunden hätte. Obwohl der Entwurf der Wohnung des Observators nur einer von vielen nicht umgesetzten Entwürfen von Universitätsgebäuden

25 Friedrich G. W. Struve, Vorläufiger Bericht von der russischen Gradmessung, mit allerhöchster Genehmigung auf Veranstaltung der Kaiserlichen Universität zu Dorpat, während der Jahre 1821 bis 1827 in den Ostseeprovinzen des Reichs (Dorpat: J. C. Schünmann, 1827).

26 Tõnu Viik, Janet Laidla, Viljar Valder, Lea Leppik, ,Esimesed katsetused Tartus“, Tart ähetorn = Tartu Old Observatory, hrsg. von Lea Leppik (Tallinn: Aasta Raamat, 2011), 27. blieb, verdient Carl Julius Senff auf jeden Fall einen Platz in der Geschichte der deutschbaltischen und der hiesigen universitären Architektur- und Kunstgeschichte am Anfang des 19. Jahrhunderts. 
Anu Ormisson-Lahe, Kristima Tiideberg: Carl Julius Senff (1804-1832) as an Architect and Graphic Artist. Additions to the Building History of the University of Tartu Ensemble Keywords: Carl Julius Senff; University of Tartu's Old Observatory; Baltic German art; university architecture

\section{CV}

Anu Ormisson-Lahe (b. 1984) is a PhD student in the Department of Art History at the University of Tartu and also works since 2010 as an Assistant of Art History at the University of Tartu, Estonia. Her main research interest is university architecture at the time of enlightenment. She has published several books about architect Johann Wilhelm Krause's work.

Kristiina Tiideberg (b. 1985) graduated with an MA in Art History from the University of Tartu in 2009; from 2008 to 2013, worked as the Keeper of the Collection of Graphic Arts at the University of Tartu Library; and from 2013 to 2019 as a curator at the University of Tartu Museum. Her research work is focused on 19th century Baltic German Graphic Arts. 